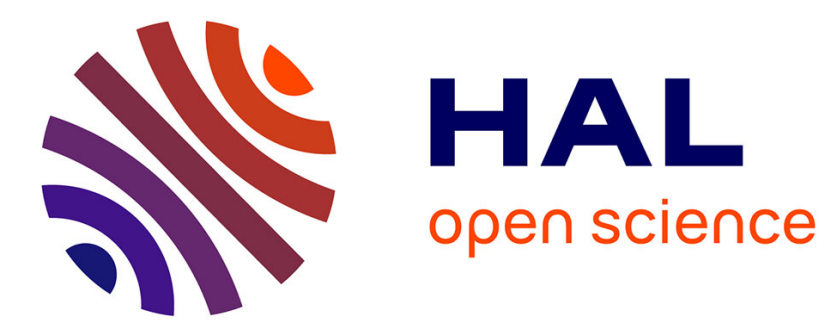

\title{
La réception américaine de Frame Analysis d'Erving Goffman
}

\author{
Nathalie Heinich
}

\section{To cite this version:}

Nathalie Heinich. La réception américaine de Frame Analysis d'Erving Goffman. Revue française de sociologie, 2019, 60 (2), pp.225-237. 10.3917/rfs.602.0225 . hal-03221667

\section{HAL Id: hal-03221667 \\ https://hal.science/hal-03221667}

Submitted on 11 May 2021

HAL is a multi-disciplinary open access archive for the deposit and dissemination of scientific research documents, whether they are published or not. The documents may come from teaching and research institutions in France or abroad, or from public or private research centers.
L'archive ouverte pluridisciplinaire HAL, est destinée au dépôt et à la diffusion de documents scientifiques de niveau recherche, publiés ou non, émanant des établissements d'enseignement et de recherche français ou étrangers, des laboratoires publics ou privés. 
«La réception américaine de Frame Analysis d'Erving Goffman »

Nathalie HEINICH

CNRS, CRAL-EHESS

96 Bd. Raspail, 75006 Paris

heinich@ehess.fr 


\section{LA RẺCEPTION AMÉRICAINE DE FRAME ANALYSIS D’ERVING GOFFMAN}

Frame Analysis (FA) est un ouvrage tardif dans l'œuvre d'Erving Goffman (Goffman, 1974) : publié en 1974, il sera suivi de Gender Advertisements en 1979 et de Forms of Talk en 1981, un an avant le décès de Goffman. Ses précédents ouvrages - beaucoup plus connus s'échelonnent sur un court laps de temps, de 1959 pour The Presentation of Self in Everyday Life à Behavior in Public Places en 1963, en passant par Asylum, Encounters et Stigma. C'est là, déjà, l'indice d'un statut un peu à part de FA dans l'ensemble de l'œuvre. Mais sa singularité tient aussi, nous allons le voir, à la nature même de cette entreprise intellectuelle.

Celle-ci est difficilement résumable par son titre : elle consiste à expliciter les structures sous-jacentes d'organisation, de perception et de catégorisation des actions humaines, selon un modèle entièrement systématisé ( «grammatical », pourrait-on dire), qui l'inscrit dans une inspiration foncièrement structuraliste, à l'opposé du courant interactionniste dont Goffman était considéré comme l'un des principaux représentants ${ }^{1}$. C'est, pour l'essentiel, ce qui lui a valu lors de sa parution d'âpres critiques, comme nous allons le voir à travers l'analyse de la douzaine de comptes rendus ou commentaires parus dans les années suivant sa publication dans diverses revues de sciences sociales (American Sociological Review, Contemporary Sociology, Human Studies, Qualitative Research, Social Research, Theory and Society...) ou ouvrages collectifs.

Au-delà du cas particulier de FA, ces débats ont ceci d'intéressant qu'ils reposent, plus ou moins explicitement, la question du choix théorique entre une perspective centrée sur les actions individuelles, dont l'interactionnisme est le meilleur représentant, et une perspective centrée sur les structures qui sous-tendent ces actions, le plus souvent à l'insu des acteurs. C'est ainsi qu'on voit se profiler, derrière les débats ayant accompagné ce livre, une réplique américaine du conflit entre le déterminisme structuraliste et la liberté individualiste, au moment où en France ce conflit rejouait, autour de l'affrontement entre sociologie bourdieusienne et sociologie boudonienne, celui qui avait agité après-guerre le monde intellectuel, partagé entre marxisme et existentialisme. Il vaut donc la peine de reconstituer cet épisode de l'histoire de la sociologie, car il soulève des questions théoriques récurrentes dans l'histoire mondiale des sciences sociales.

Nous ne tenterons pas toutefois ici une généalogie intellectuelle de l'œuvre de Goffman, dans la tradition de l'histoire des idées : il ne s'agira pas d'entrer dans les débats

\footnotetext{
${ }^{1}$ Pour une présentation du livre à l'usage des lecteurs francophones (l'ouvrage a été traduit en français en 1991, aux éditions de Minuit), cf. Heinich, 1991 ; Cefaï et Gardella, 2012.
} 
relatifs à la place de cet auteur dans les sciences sociales, ni même à l'influence exercée par tel ou tel courant sur Frame Analysis mais, beaucoup plus modestement, d'observer la façon dont cet ouvrage a été reçu, et de comprendre les raisons de cette réception conflictuelle.

\section{La critique de Denzin et Keller}

Dès sa parution, et dans les années qui suivirent ${ }^{2}$, le livre suscita une réception étonnamment controversée pour un chercheur aussi reconnu que Goffman l'était à l'époque. Partagés entre déception et enthousiasme (Craib, 1978, p. 79), les comptes rendus du livre ne se contentèrent pas d'en expliciter les antécédents, notamment dans la tradition philosophique (William James sur la définition du sens de la réalité : Manning, 1980) et anthropologique (Gregory Bateson pour l'introduction du terme de « cadre » dans les sciences sociales : Manning, 1980) : c'est un véritable conflit d'interprétations et, au-delà, une lutte de clans sociologiques, qui éclata à cette occasion.

Le compte rendu le plus remarqué fut très critique : fustigeant une orientation jugée trop « structuraliste » et insuffisamment « interactionniste », il provoqua une intéressante réplique de Goffman lui-même, pourtant peu enclin à ce type de polémiques. Publié dans Contemporary Sociology, il était dû à deux sociologues, Norman Denzin (spécialiste de recherche qualitative) et Charles Keller (Denzin \& Keller, 1981). Ils proposent tout d'abord une intéressante caractérisation du structuralisme, en huit points : l'activité sociale est supposée être régulée par un ensemble de règles présentes à un niveau profond ; ces règles se déploient en surface, au niveau de la vie quotidienne ; les comportements sont analysables comme une totalité, où priment les relations de toutes sortes, y compris les règles de parenté et les mythes; il est possible de réduire ces relations à un système d'oppositions binaires ; ce système d'oppositions binaires est basé à la fois sur des oppositions logiques et sur la perception des différences ; les configurations structurales se modifient en se transformant en d'autres structures, à travers des règles de transformation ; les significations, les motifs, les intentions sont des processus incorporés dans les structures et non dans les personnes, de sorte que ce sont les structures, les codes, les cadres qui déterminent le comportement humain, selon une organisation syntaxique que la théorie explicite à travers des lexiques, des clés, des codes ; les présentations structuralistes sont hiérarchisées et prennent la forme d'une arborescence (Denzin \& Keller, 1981, p. 54).

\footnotetext{
${ }^{2}$ Les commentaires de l'œuvre ne se limitèrent pas aux années suivant immédiatement sa parution : dans les années 2000 apparurent aussi des tentatives pour automatiser le codage des «cadres » goffmaniens : cf. Koenig, 2006 ; Sanfilippo et al., 2008.
} 
Aux yeux de Denzin et Keller, Frame Analysis correspond en tous points à cette caractérisation du structuralisme. Le problème est que, pour eux, il s'agit là d'une sociologie antinomique de celle que non seulement ils pratiquent et promeuvent mais de celle qui aurait été jusqu'alors, également, la sociologie de Goffman. Ainsi ce livre constituerait une trahison de la tradition interactionniste qui leur aurait été commune, et notamment de sa dimension interprétative héritée de James, de Schutz, de Mead, de Cooley, de Weber, « basée sur la subjectivité, le sens, l'intention », et qui «étudie l'interaction sociale comme un processus, une série d'événements qui en se produisant transforment le courant phénoménologique propre à chaque acteur ». Dans cette perspective, ce sont « les individus doués de réflexivité, de sentiments, de capacités d'interaction qui sont au cœur du paradigme interprétatif », de sorte que «le sens doit être trouvé dans l'interaction, et non dans les structures ou les règles » (Denzin \& Keller, 1981, p. 53), ainsi que dans les « réalités multiples des différents individus présents dans une situation » (Denzin \& Keller, 1981, p. 59).

A l'opposé, Frame Analysis s'inscrirait dans la tradition aristotélicienne de la catégorisation objectiviste plutôt que du processus interprétatif, telle la biologie linéenne (Denzin \& Keller, 1981, p. 56), et délaisserait la recherche de sens au profit de la quête d'un système (Denzin \& Keller, 1981, p. 57). D'où une critique dont la radicalité surprend à propos d'un auteur aussi reconnu que l'était Goffman à l'époque : «Ses cadres sont des formes gelées. Sa conception de la réalité est illusoire et floue. Les transformations telles qu'il les postule ne proviennent d'aucune cause ni d'aucun processus. Ses cadres n'attrapent que des événements situés à la périphérie de la vie quotidienne. (...) Ses acteurs sont des monades, isolés dans des cadres et regardant le monde extérieur. Il n'y a pas d'interaction dans FA. Les sujets sont relégués sur les bas-côtés. Ils ne sont pas nécessaires au projet goffmanien » (Denzin \& Keller, 1981, p. 59).

\section{La réponse de Goffman}

Erving Goffman publia dans le même numéro de Contemporary Sociology une réponse à la fois ferme et fournie (Goffman, 1981) : sans doute était-il particulièrement soucieux de défendre celui de ses livres qui pouvait le mieux prétendre au statut théorique dont dépend le prestige en sciences sociales (Berger, 1986, p. XII-XIII). « Il n'y a vraiment pas grand-chose dans cet essai de Denzin et Keller avec quoi je suis d'accord ; et il n'y a rien à quoi je trouve un mérite quelconque », affirme-t-il sans prendre de gants (Goffman, 1981, p. 62). 
Il s'y démarque à la fois du structuralisme « à la française » et d'une conception trop systématique de l'interactionnisme. Concernant, donc, le structuralisme :

«Les auteurs semblent se référer à la version courante, associée principalement aux auteurs français. Je dois avouer que ces textes me laissent tout aussi perplexes qu'eux. La notion d'opposition binaire me paraît avoir du sens dans les termes initialement posés par l'école de Prague dans son approche de la phonologie, mais la façon dont Lévi-Strauss et d'autres ont adapté et étendu cette notion évoque la dialectique hégélienne et autres tours de passe-passe verbaux, tout à fait étrangers à l'empirisme de base dans lequel j'ai été élevé. Le terme de «structure » au sens où je l'entends est repris aux analyses traditionnelles (à présent très critiquées) des systèmes de parenté, et bien que je trouve très plaisante la lecture de Roland Barthes, je dois mon orientation vers l'étude des matériaux de fiction à l'enseignement de Berelson sur l'analyse de contenu (il y a de cela quelques années), et aux premières recherches en langue anglaise sur la culture populaire, à savoir celles de George Orwell et Dwight Macdonald. Quant à ma conception de la transformation, je la dois à la lecture de Growth and Form (1942) de D'arcy Thompson - un structuraliste qui n'est français que par son prénom » (Goffman, 1981, p. 62).

Concernant, par ailleurs, l'interactionnisme, il en récuse fermement certaines dérives outrancières : «Les individus auxquels j'ai affaire n'inventent pas le jeu d'échecs chaque fois qu'ils s'assoient pour jouer ; ils n'inventent pas davantage le marché financier quand ils achètent un titre quelconque, ni le système de la circulation piétonne quand ils se déplacent dans la rue. Quelles que soient les singularités de leurs motivations et de leurs interprétations, ils doivent, pour participer, s'insérer dans un format standard d'activité et de raisonnement qui les fait agir comme ils agissent »(Goffman, 1981, p. 62). Il se démarque également de l'usage psychologisant que faisait Bateson avant lui du terme de «cadre » : «Il faisait du cadrage un processus psychologique, alors que je le vois comme inhérent à l'organisation des événements et de l'activité cognitive » (Goffman, 1981, p. 64).

Bref, il réaffirme haut et fort sa conception du système de cadres comme une structure d'arrière-plan définissant les conditions de possibilité des interactions étudiées par ses collègues se réclamant du courant dont lui-même est issu : comme il l'avait affirmé dans son livre, « la société est bien une structure de cadres » (« a framework of frames »).

\section{L'interprétation structuraliste}

Mais Goffman s'est-il vraiment détourné de l'interactionnisme pour adopter, dans Frame Analysis, le positionnement structuraliste ? Une autre interprétation est possible, qui revisite l'œuvre de Goffman en montrant qu'il n'a peut-être pas été, dès le début, 
l'interactionniste qu'on croit, et qu'il existe une continuité entre ses premiers ouvrages et Frame Analysis (Collins, 1988). Cette position avait été défendue, avant la critique de Denzin et Keller et la réponse de Goffman, dans un article de George Gonos publié dans American Sociological Review trois ans après la sortie du livre. Il y oppose « situation » et «frame »: selon lui, on comprend mieux l'ensemble de l'œuvre de Goffman si on la voit comme relevant non de l'interactionnisme symbolique, mais du structuralisme (Gonos, 1977).

Pour Gonos, l'approche interactionniste s'appuie principalement sur la notion de « situation », qui prend sa source dans la sociologie de Max Weber, tandis que la notion de « cadre » s'inscrit dans une tradition structuraliste héritée d'Émile Durkheim (Gonos, 1977, p. $855)^{3}$. Ainsi les concepts de « situation » et de « cadre » impliquent « des approches bien différentes de la place de l'analyse individuelle. Les interactionnistes mettent l'accent sur les capacités de construction du monde mobilisées par les acteurs dans les situations quotidiennes et y voient la source du changement social, tandis que le concept de cadre amène Goffman à considérer les individus comme de simples « supports » de l'existence continue des structures sociales »(Gonos, 1977, p. 866).

Corrélativement, dans la perspective interactionniste initiée par George Mead c'est le sujet individuel qui est l'unité première, élémentaire, existant avant les structures sociales (Gonos, 1977, p. 864) ; or Goffman, y compris dans La Présentation de soi dans la vie quotidienne, aurait «miné l'appui de la micro-sociologie américaine sur les sujets individuels » (Gonos, 1977, p. 865) : son œuvre - et en particulier Frame Analysis constituerait une « attaque contre le sujet, c'est-à-dire contre la conscience individuelle » (Jameson, 1976, p. 120). En d'autres termes, « ce projet, qui s'annonce dès lors comme une critique de la notion de définition de la situation, sera formalisé dans Frame Analysis, c'est-àdire après que Goffman aura déchargé l'interactionnisme de son fardeau le plus encombrant : le sujet »(Ogien, 1989, p. 104).

Par ailleurs, contrairement au courant interactionniste, la sociologie de Goffman devrait être vue selon Gonos comme une « sociologie formelle », c'est-à-dire comme une tentative pour abstraire de la vie quotidienne un nombre limité de formes ou de modes d'existence (Gonos, 1981, p. 855 et Jameson, 1976, p. 119). Cette orientation formaliste renverrait, elle, vers une tradition remontant à Georg Simmel, selon Peter Manning (Manning, 1980, p. 269). Du même coup Frame Analysis - le plus «formel », à n'en pas douter, des

\footnotetext{
${ }^{3}$ Cette parenté de toute l'œuvre de Goffman avec la tradition durkheimienne sera également affirmée par Peter Manning, selon qui la vie sociale est vue par lui comme extérieure aux individus, contraignant leurs actions et existant avant et après l'existence individuelle (Manning, 1980, p. 262).
} 
ouvrages de Goffman - ne peut pas reposer sur le même type d'exploitation des données qu'une analyse des situations (Gonos, 1981, p. 857), dont l'unicité appelle la minutie des descriptions empiriques (Gonos, 1981, p. 855). Il s'agit bien plutôt de dégager les règles des cadres de manière « analogue aux structures syntaxiques du langage » (Gonos, 1981, p. 859) et l'on retrouve là, bien sûr, une propriété ancrée dans la tradition structuraliste. Le rôle du sociologue devient dès lors de déchiffrer « un code, un système inconscient de catégories et de règles gouvernant la création et la communication du sens » (Gonos, 1981, p. 863) ${ }^{4}$. Et l'objet du livre n'est pas tant la réalité sociale que le système exposé par le livre lui-même, et dont il s'agit de tester la validité (Jameson, 1976, p. 128), dans une auto-référentialité qui l'inscrit pleinement dans le genre grammatical.

Une tout autre méthodologie est donc requise, qui ne repose pas sur la perspective compréhensive d'explicitation du vécu par le sujet lui-même, visant «le sens attaché aux situations par les acteurs », que les interactionnistes considèrent comme « la source de données la plus importante » : c'est que les règles telles que les conçoit le structuralisme « ne peuvent se découvrir dans la conscience des participants, par l'intersubjectivité », de sorte que «Goffman reste profondément convaincu de l'inutilité de prendre en compte la subjectivité des acteurs, se méfiant même particulièrement de l'énonciation verbale de leurs motivations » (Gonos, 1981, p. 864). Autant l'analyse d'une situation à travers la subjectivité des acteurs passe par la documentation des états intérieurs à travers des questionnaires, des entretiens, voire de simples dialogues, dans la droite ligne de la méthode wébérienne du Verstehen (Gonos, 1981, p. 864), autant l'analyse grammaticale de l'expérience, en s'intéressant prioritairement aux «propriétés non intentionnelles et non conscientes »du comportement des acteurs, s'éloigne de la sociologie compréhensive, comme l'ont noté Daniel Cefaï et Edouard Gardella $^{5}$ (Cefaï \& Gardella, 2012, p. 235-236) - du moins tant qu'on conçoit celle-ci comme une mise en évidence de « représentations » qui guideraient consciemment les acteurs (Cefaï \& Gardella, 2012, p. 256), à l'exclusion de ce qui demeure dans l'implicite voire dans le non conscient.

\section{Un autre paradigme}

\footnotetext{
${ }^{4}$ Cette dimension sémiotique ou grammaticale du projet cadre-analytique a été soulignée également par F. Jameson, 1976, p. 119-120.

${ }^{5}$ C'est pourquoi aussi, expliquent-ils, « l'analyse de cadres ne porte pas sur des extraits de textes déjà refroidis, mais se propose de ressaisir des énonciations de paroles en contexte, sur le vif. Les énoncés débordent alors leur simple contenu lexical et font émerger des propriétés pragmatiques, riches en inférences, qui sont autant de supports pour l'analyse des opérations de cadrage » (Cefaï et Gardella, 2012, p. 251).
} 
Au terme de cet autre éclairage de l'œuvre de Goffman, à la lumière de la perspective structuraliste, la conclusion s'impose : son interactionnisme a été largement surestimé et, avec lui, sa dette envers Mead, comme le précisera Peter Manning : «Bien que Goffman ait adressé quelques signes polis en direction de l'interactionnisme symbolique, citant favorablement Mead et Blumer, sa dette principale va à la sociologie formelle - celle de Mauss, de Durkheim, de Simmel - et, plus récemment, doit se chercher dans son affection pour la sémantique structurale, qu'il se plaît à citer. (...) L'accent mis par Mead sur les sujets individuels plutôt que sur les contraintes imposées à l'interaction par les formes sociales dans lesquelles elle se produit, sur lesquelles Goffman insiste même dans des ouvrages antérieurs à Frame Analysis, marque la différence entre les perspectives meadienne et goffmanienne » (Manning, 1980, p. 271).

Frame Analysis devient ainsi une sorte de miroir grossissant d'un structuralisme latent qui habiterait aussi le reste de l'œuvre, derrière un intérêt pour les situations que Goffman partage avec l'interactionnisme (même si elles ne sont pour lui qu'un appui méthodologique et non pas un objet) : un intérêt commun pour les situations qui rompait avec une macrosociologie longtemps dominante, notamment dans la tradition parsonienne. Les cadres sont donc bien des structures « au sens de Piaget, Althusser et Balibar » (Gonos, 1981, p. 860), et si Goffman s'intéresse aux interactions, c'est pour y découvrir les règles implicites qui, en «définissant la situation », les gouvernent (Berger, 1986, p. XIII). S'inscrivant pleinement dans le paradigme structuraliste, cet ouvrage le renouvelle par son application non à des représentations symboliques (œuvres d'art, mythes...) mais à la vie quotidienne (Gonos, 1981, p. 858) - d'autres diront même, plus radicalement, à « la structure de la réalité » (Garcia Landa, 2016).

Certes, la réalité qu'il prend pour objet n'est pas, aux yeux de ses opposants, suffisamment quotidienne : «On n'y trouve pas d'analyse de ce qu'ils [les acteurs] font, tous les jours : se saluer, se faire des adieux, affirmer leurs relations, aller se coucher, se lever, raconter des histoires, se promener, bavarder, engager des conversations », regrettent Denzin et Keller (Denzin \& Keller, 1981, p. 320). En termes cadre-analytiques, ils déplorent que les « cadres primaires » aient été un peu trop délaissés au profit des «cadres transformés » (et notamment du cadre théâtral), dont ils estiment qu'ils ne constituent que « les aspects périphériques de l'expérience quotidienne »(Denzin \& Keller, 1981, p. 320). Ce à quoi Goffman aurait pu leur répondre que cette focalisation sur les transformations de cadres a des raisons avant tout méthodologiques : ce sont les changements qui font apparaître les règles implicites, tandis que les cadres à l'état stable, surtout lorsqu'ils ont la simplicité des «cadres 
primaires », ne sont pas de bons révélateurs des structures sous-jacentes. C'est pour les mêmes raisons que, dans d'autres domaines, l'analyse des controverses, des tensions, des problèmes rencontrés par les acteurs deviendra, pour la sociologie pragmatique, un outil sociologique de premier ordre (Lemieux, 2018).

Le livre peut donc engendrer des désaccords sur la définition même de la réalité : à la réalité quotidienne privilégiée par l'interactionnisme symbolique, il préfère ces « réalités non officielles ou secondaires, ces interactions secrètes ou ces fictions provisoires », auxquelles est accordée une sorte de «priorité ontologique », de sorte que «l'expérience authentique se définit comme telle entre les actes, et derrière les scènes dans le théâtre de la vie quotidienne » (Garcia Landa, 2016, p. 6). Dès lors ce ne sont donc pas seulement les notions d'individu et de société qui passent au second plan, mais « la notion même de réalité quotidienne en tant que réalité première, fondamentale »(Davis, 1975, 599). Car si ce qui intéresse Goffman n'est pas l'exceptionnalité mais la banalité, celle-ci ne l'intéresse pas dans sa quotidienneté, comme les interactionnistes, mais dans ce qui, à travers elle, se joue en-deçà de l'expérience : cette grammaire partagée qui, justement, permet de la rendre banale aux yeux des acteurs. Comme le dit son préfacier dans une formule ramassée, l'auteur de Frame Analysis est un «parfait métaphysicien du banal » (Berger, 1986, p. XI).

En changeant de paradigme - passant de l'interactionnisme au structuralisme -, c'est toute la perception de l'ouvrage (et, au-delà, de l'œuvre entière de Goffman) qui bascule : comme si l'on passait de la forme-lapin à la forme-canard, pour reprendre une image familière à la psychologie de la forme. Mais c'est aussi son évaluation qui change radicalement de sens, amenant à interpréter ce canard comme le célèbre « vilain petit canard » d'Andersen : perçu dans la catégorie « canard», il est moche, raté, indigne de sa famille d'appartenance, et donc moqué, stigmatisé, rejeté ; mais dès lors qu'en grandissant il donne prise à une perception conforme à sa véritable catégorie, c'est un cygne qui se révèle, magnifique et majestueux...

\section{Un livre déroutant}

Voilà qui est « déroutant », bien sûr : « C'est ce mixte original entre "structuralisme" et "interactionnisme" qui continue de dérouter à la lecture de Goffman » (Cefaï \& Gardella, 2012, p. 234). Et le plus déroutant n'est pas le silence de la cadre-analyse sur les différences de classes, auxquelles Goffman ne s'intéresse ici nullement (Hazelrigg, 1992, p. 239) alors que, depuis la montée en puissance de la sociologie bourdieusienne, elles sont devenues l'alpha et l'omega de la discipline pour une grande partie de ses praticiens. Le plus déroutant n'est pas non plus la faible présence d'une mise en perspective historique (quoique, comme la 
question de la stratification sociale, rien n'empêcherait de l'introduire dans le modèle), selon le reproche classique fait à toute entreprise structuraliste, et qui n'a pas manqué d'être adressé à Goffman (Sharron, 1981, p. 500) - lequel protestera : «Les cadres sont sujets à variations historiques, ce qui revient fréquemment dans FA même si j'en donne peu de détails étant donné ma monumentale ignorance en la matière » (Goffman, 1981, p. 64).

Non : le plus déroutant demeure cette difficulté à inscrire le livre dans les catégories épistémiques consacrées dès lors qu’on a « cadré » la sociologie goffmanienne comme appartenant à l'interactionnisme : «Toute la difficulté de Goffman se joue là et interdit les lectures de type "interactionniste" ou "structuraliste" »(Cefaï \& Gardella, 2012, p. 260). Si, pour lui comme pour les interactionnistes, la définition de la réalité est relative à la situation, il s'agit chez lui d'un « relativisme modéré » (Nizet \& Rigaux, 2005, p. 81), car figure toujours à l'arrière-plan le système organisateur de la situation. Et si, pour lui, « l'ordre de l'interaction constitue un domaine d'enquête de plein droit », doté de « ses propres lois », «ce n'est pas tant pour faire de celle-ci son objet qu'afin de s'opposer au fonctionnalisme dominant, centré sur les régulations institutionnelles que les individus ne feraient qu'appliquer pour produire l'ordre social » (Cefaï \& Gardella, 2012, p. 241).

Cette relecture oblige à admettre que dans toute son œuvre, Goffman associe certaines propriétés de l'interactionnisme avec le schème de pensée structuraliste : crime de lèsefrontières entre catégories épistémiques supposées incompatibles. Pire : il associe ces deux polarités antinomiques que sont le «micro » et le « macro », l'observation rapprochée des situations et l'explicitation des règles les plus générales qui les organisent. C'est la thèse que soutient Wendy Leeds-Hurwitz : «Le concept d'ordre social est la clé de la théorie goffmanienne. (...) L'œuvre de Goffman opère sur deux niveaux, celui de la macroanalyse (l'ordre social) et celui de la microanalyse (l'interaction) ; s'il est connu prioritairement pour ce dernier, c'est dans le premier qu'il développe sa théorie. Et c'est la compréhension de la relation entre les deux qui permet de pleinement comprendre l'œuvre de Goffman » (LeedsHurwitz, 1986). Bennet Berger a lui aussi repéré ce mélange atypique : une « attention à la microstructure des significations engendrées par de petites actions routinières », ainsi qu'une «stricte rigueur ethnographique dans la description exacte des comportements » (Berger, 1986, p. XII), combinées avec «l'influence de la tradition macrosociologique d'Emile Durkheim, assimilée via le fonctionnalisme de l'anthropologie sociale britannique » (Berger, 1986, p. XV).

Goffman lui-même revendique à la fois le primat de la perspective macrosociale et le droit à l'aborder à travers cet aspect « secondaire » qu'est l'expérience individuelle : «Ce 
livre traite de l'organisation de l'expérience - non de l'organisation de la société (...). Je ne m'y occupe pas de la structure de la vie sociale mais de la structure de l'expérience que les individus ont à tous moments de leur vie sociale. Je considère personnellement que c'est la société qui vient en premier à tous points de vue, et que tout ce qui concerne l'implication individuelle ordinaire vient en second; cet ouvrage ne traite que de sujets seconds » (Goffman, 1981, p. 65). Ne faut-il pas voir là une incitation à dépasser l'opposition agonistique entre micro- et macro-sociologie, pour les envisager comme les pôles opposés sur un continuum de possibilités méthodologiques adaptées à la nature des objets et des problématiques?

\section{Une querelle à double fond}

Mais au fait, pourquoi cette requalification de Goffman en apostat de l'interactionnisme, et de sa sociologie en vitrine du structuralisme, a-t-elle fait couler autant d'encre et suscité autant de critiques ?

Une réponse nous est suggérée par la remarque de Bennett Berger à propos du « modèle de lien entre microsociologie et macrosociologie » que fournit Frame Analysis : « Nous préférons considérer les relations en face-à-face comme l'un des derniers refuges de chaleur au sein de l'hiver bureaucratique. Sous l'objectif de Goffman, nous découvrons une micropolitique aussi sinistrement glaciale et aussi joyeusement organisée que la diplomatie internationale » (Berger, 1986, p. XVIII). En d'autres termes, et quitte à caricaturer un peu, l'on pourrait résumer ainsi l'opposition entre les deux écoles : la perspective interactionniste nous rendrait sensibles à la chaleur des relations humaines, tandis que la perspective structuraliste nous soumettrait à la rigueur abstraite du déterminisme.

Plus encore : l'interactionnisme serait la garantie d'une attention proprement morale au sujet individuel, opposé à « la société ». C'est du moins ce qu'avança Eliot Freidson dans sa nécrologie de Goffman : «Les premiers ouvrages de Goffman prennent pour objet le sujet individuel, dans un monde qui le crée et l'opprime à la fois. Son œuvre est intensément morale, marquée par une défense passionnée du sujet contre la société. (...) Ce qui lui confère une valeur qui durera bien plus longtemps que d'autres sociologies, c'est son intense humanité individuelle, et son style » (Freidson, 1983, p. 359). Or rien dans cet éloge n'est plus éloigné, assurément, de la cadre-analyse, et probablement aussi du reste de l'œuvre de Goffman, caractérisée par la passion épistémique pour la compréhension de l'expérience humaine beaucoup plus que par la compassion humaniste pour la défense du sujet opprimé par la société - pour caricaturer, à peine, ce lieu commun de l'individualisme. 
Et, pas davantage que par un projet moral, l'œuvre goffmanienne n'est-elle habitée par un projet politique, au grand dam de ceux qui voudraient voir dans l'interactionnisme une façon détournée de s'engager dans la grande cause du sujet en butte au « social». Car, comme le souligne William Gamson, Goffman «ne s'intéressait pas à l'analyse de l'interaction dans le but d'apprendre de quelle façon elle contribue à la mobilisation collective dirigée vers le changement social. Il ne s'intéressait pas aux transformations de la conscience politique, ni à la façon dont les médias et autres institutions sociales les rendent si difficiles » (Gamson, 1985, p. 622) - hélas pour les partisans de la sociologie critique. Ni progressiste ni conservateur, comme le souligne Bennett Berger, « il n'avait guère de goût, contrairement aux intellectuels progressistes, pour l'indignation morale contre les injustices des règles de cadrage ou les inégalités des bénéfices qu'elles procurent. Mais il avait encore moins le respect des règles propre aux conservateurs » (Berger, 1986, p. XVI).

Il faut bien le reconnaître : la sociologie de Goffman - et tout particulièrement Frame Analysis - ne nous fournit aucune arme pour équiper la liberté du sujet contre le poids des déterminations sociales. Et c'est bien là, probablement, qu'il faut chercher le fond de l'indignation suscitée par l'inflexion cadre-analytique de son œuvre : la querelle n'est pas seulement épistémique - visant les outils de la production de connaissance - mais aussi, implicitement, politique et morale, visant une cause humaniste à défendre.

Dans l'un de ses ouvrages les plus proches de l'interactionnisme, Encounters, Goffman soupçonnait dans ce courant une volonté implicite de «maintenir une partie du monde à l'abri de la sociologie ». C'est dire qu'en s'en éloignant - ou, plutôt, en manifestant qu'il n'avait jamais vraiment appartenu à cette famille qui pourtant le revendiquait comme l'un de ses membres les plus éminents - il ne faisait que suivre la pente qui, depuis toujours, l'avait attiré vers la sociologie. La conclusion s'impose : plus encore que tout le reste de son œuvre, Frame Analysis représente, selon les mots de Lawrence Hazelrigg, « le summum de la sociologie $»^{6}$.

\section{Du bon usage des théories}

A la fin de sa réponse à ses contradicteurs Denzin et Keller, Goffman se livre à une consistante auto-critique de son propre livre : il lui reproche notamment de trop s'appuyer sur de la fiction et des articles de journaux (Frame Analysis est essentiellement construit sur

\footnotetext{
${ }^{6}$ « Pour de tels lecteurs, l'argument de Goffman est dérangeant non parce qu'il soumettrait les capacités d'action des acteurs à quelque abstraite structure "structuraliste" (ce n'est pas le cas), mais parce qu'il est si implacablement, même si c'est de façon différente, sociologique. On pourrait même dire qu'il manifeste le summum de la sociologie" (Hazelrigg, 1992, p. 264).
} 
l'analyse de faits-divers, pour la plupart empruntés au San Francisco Chronicle), de ne pas avoir assez détaillé les cadres primaires ainsi que les passages entre cadres, ou encore d'avoir laissé passer quelques ambiguïtés terminologiques (Goffman, 1981, p. 67). Mais ces défauts ne justifient pas à ses yeux la tentative de disqualification radicale dont il a fait l'objet. En revanche, ses adversaires théoriques trahissent, eux, une conception du travail scientifique autrement plus problématique à ses yeux. C'est sur cette leçon de conduite épistémologique que nous conclurons.

Ses détracteurs, selon lui, « ont des paradigmes à mettre en pièces, et une large perspective à défendre et à promouvoir. Mais le vif intérêt qu'ils entretiennent ainsi pour certains livres est entravé chez eux par cela même dont ils m'accusent: à savoir une conception fort rigide de la réalité sociale » (Goffman, 1981, p. 68).

Fustigeant «le ton de la dénonciation théologique ou politique » employé dans ce qui devrait demeurer une discussion scientifique, il résume ainsi leur posture intellectuelle : «On proclame son appartenance à une perspective donnée, on mentionne pieusement ses textes fondamentaux, et on annonce que l'auteur en question en est exclu faute de présenter les qualités requises pour y appartenir. Une affaire d'étiquetage. Comme si un ouvrage était un objet unitaire et pouvait être totalement mauvais parce que l'auteur ne semble pas souscrire à telle doctrine, laquelle doctrine, si l'on y souscrivait, rendrait l'ouvrage bon. (...) J'admets que des étudiants en sociologie puissent éprouver le besoin de tels formulaires idéologiques (analogue au besoin d'écoles de pensées ou de "paradigmes"), pour pouvoir montrer à leur examinateur qu'ils ont des convictions sociologiques et un certain sens de la sociologie en tant que champ, et j'admets que leurs enseignants aient recours aux mêmes mots d'ordre pour établir leur réputation dans la salle de classe ; mais je trouve attristante la tendance récente à tirer des publications à partir de ce genre de besoins. » (Goffman, 1981, p. 61) ${ }^{7}$.

En d'autres termes, Goffman revendique un usage instrumental des concepts et des théories, plutôt que l'usage catégorisant pratiqué par ses adversaires : eux s'intéressent aux théories avant tout en tant qu'elles marquent des appartenances, des territoires conceptuels, des familles de pensées ; lui ne veut y voir que des outils, utilisables au gré des besoins du chercheur en fonction de ses objets, de ses problématiques, de ses visées. Plutôt que la lutte des clans théoriques qu'affectionnent ses adversaires, lui s'intéresse avant tout à sa propre

\footnotetext{
${ }^{7}$ Il leur reprochera également de prétendre enfermer sa propre conception du « cadre » dans celle de Bateson : « Mais suis-je obligé, pour qu'on ajoute foi à ce que je dis, de n'emprunter à Bateson (...) que ce que D. et K. semblent y trouver? Et faut-il que, m'inspirant de Bateson, je n'éprouve pas de désaccord avec lui ? Bateson a conçu le cadrage comme un processus psychologique ; pour moi, au contraire, il est inhérent à l'organisation des événements et à la connaissance ». (Goffman, 1981, p. 61).
} 
pensée, et aux façons les plus efficaces de la développer et de la mettre à l'épreuve, dans le but de mieux rendre compte de ses objets et non pas de revendiquer des affiliations.

Les dogmes ne sont-ils pas faits pour être bousculés par les objets auxquels on tente de les appliquer, et les paradigmes pour être remplacés? 


\section{BIBLIOGRAPHIE}

Berger B., 1986, Foreword to Frame Analysis, Boston, Northeastern University.

Cefaï D. \& Gardella E., 2012, « Comment analyser une situation selon le dernier Goffman ? De Frame Analysis à Forms of Talk», in Cefaï D. et Perreau L. (éds.), Erving Goffman. L'ordre de l'interaction, Paris, CURAPP-PUF.

Collins R., 1988, “Theoretical Continuities in Goffman's work”, in Drew P. \& Wotton A. (eds.), 1988, Erving Goffman: Exploring the Interaction Order, Cambridge, Polity Press.

Craib I., 1978, «Erving Goffman: Frame Analysis”, Philosophy of the Social Sciences, 8, 4, pp. 79-86.

Davis M., 1975, Review of Frame Analysis, Contemporary Sociology, 4, 6, pp. 599-603.

Denzin N., Keller Ch., 1981, “Frame Analysis Reconsidered”, Contemporary Sociology, 10, 1, pp. 52-60.

Freidson E., 1983, “Celebrating E. Goffman”, Contemporary Sociology, 13, 4, pp. 359-362.

Gamson W., 1985, “Goffman's Legacy to Political Sociology”, Theory and Society, 14, 5, pp. 605-622.

Garcia Landa J. A., 2016, "The (In)Definition of Reality: Reframing and Contested Topsight”, February 29 (https://ssrn.com/abstract=2763243 ou http://dx.doi.org/10.2139/ssrn.2763243).

Goffman E., 1974, Frame Analysis. An Essay on the Organization of Experience, New York, Harper and Row (Les Cadres de l'expérience, Paris, éditions de Minuit, 1991, traduit par Isaac Joseph avec Michel Dartevelle et Pascale Joseph).

Goffman E., 1981, “A reply to Denzin and Keller”, Contemporary Sociology, 10, 1, p. 60-68 ( «Réplique à Denzin et Keller », traduit par Louis Quéré, in Joseph I., Castel R. \& Cosnier J. (éds.), 1989, Le Parler-frais d'Erving Goffman, Paris, Minuit).

Gonos G. 1977, “"'Situation" versus "Frame": The "Interactionist" and the "Structuralist" Analysis of Everyday Life", American Sociological Review, 42, pp. 854.

Hazelrigg L., 1992, "Reading Goffman's Framing as Provocation of a Discipline”, Human Studies, 15, 2/3, pp. 239-264.

Heinich N., 1991, «Pour introduire à la cadre-analyse », Critique, n 535, pp. 936-953.

Jameson Fr., 1976, “On Goffman's Frame Analysis”, Theory and Society, 3, 1, pp. 119-133.

Koenig Th., 2006, "Compounding mix-methods problems in frame analysis through comparative research", Qualitative Research, 1. 
Leeds-Hurwitz W., 1986, "Erving Goffman and the Concept of Social Order", communication au colloque "Erving Goffman: An Interdisciplinary Appreciation", Université de York, 8-11 juillet 1986.

Lemieux C., 2018, La Sociologie pragmatique, Paris, La Découverte, collection Repères.

Manning P., 1980, “Goffman's Framing Order: Style as Structure”, in Jason Ditton (ed.), The View from Goffman, Basingstoke, Macmillan, pp. 252-284.

Nizet J., Rigaux N., 2005, La Sociologie d'Erving Goffman, Paris, La Découverte-Repères.

Ogien A., 1989, « La Décomposition du sujet », in Joseph I., Castel R. \& Cosnier J. (éds.), 1989, Le Parler-frais d'Erving Goffman, Paris, Minuit.

Sanfilippo A. et al., 2008, “Automating Frame Analysis”, in Liu H., Salerno J., Young M. (eds.), Social Computing, Behavioral Modeling, and Prediction, Boston, Springer.

Sharron A., 1981, "Frame Paralysis: when Time stands still”, Social Research, 48, 3, 1981, pp. 500-520. 


\section{RESUME}

Frame Analysis, ouvrage tardif dans l'œuvre d'Erving Goffman, suscita à sa parution, et dans les années qui suivirent, une réception étonnamment controversée pour un chercheur aussi reconnu qu'il l'était à l'époque. Il vaut la peine de reconstituer cet épisode de l'histoire de la sociologie américaine, et de tenter d'en repérer les causes. En effet, au-delà du cas particulier de ce livre, il soulève des questions théoriques récurrentes dans l'histoire mondiale des sciences sociales, tenant notamment aux statuts respectifs de l'interactionnisme et du structuralisme et, plus généralement, à l'importance qu'il convient d'accorder aux catégorisations théoriques. 